\title{
EVALUATION OF DRAINAGE CHANNELS ON PABUARAN ROAD CIBINONG DISTRICT OF BOGOR REGENCY
}

\author{
Jantiara Eka, NANDIASA \\ Faculty of Engineering, University of Mercu Buana, Jakarta, Indonesia \\ jantiara@mercubuana.ac.id \\ Ridwan Adi, SANJAYA \\ Faculty of Engineering, University of Mercu Buana, Jakarta, Indonesia \\ Ridwanadisanjaya@gmail.com
}

\begin{abstract}
Drainage can be generally defined as a technical measure to reduce excess water, whether from rain, seepage, or excess irrigation water from an area / land, so that the function of the area / land is not disturbed. This study aims to analyze, assess and evaluate the condition and capacity of the channel in the sub-district Pabuaran Road Bogor Cibinong District to discharge the flooding. Data and information used is primary data in the form of rainfall data from the Meteorology and Geophysics from the nearest irrigation hall as well as secondary data, the authors survey directly at the sites. In the flood discharge processing method using two methods of hydrograph Unit hydrograph Unit Synthetic Synthetic Nakayasu and ITB. The results of both methods is used the largest flood discharge. Getting the design flood discharge using Synthetic Unit Hydrograph ITB on Channel A of 6,434 m3 / sec. Analysis of the results of the calculation of the existing discharge chute, the discharge contained on Channel A of $2.10 \mathrm{~m} 3 / \mathrm{sec}$ and Channel B amounting to $0.21 \mathrm{~m} 3 / \mathrm{sec}$ limpas both channels experience. Alternative solutions to the problems that limpas channel or unsafe they are planning a new dimension channel.
\end{abstract}

Keywords: Drainage, Hydrology Analysis, Debit Flood Plan, ITB HSS, HSS Nakayasu

\section{INTRODUCTION}

Drainage is one of the basic facilities that are designed as a system to meet the needs of society and is an important component in urban planning (infrastructure planning in particular). Drainage is also interpreted as a way of disposal of unwanted excess water in an area, as well as ways alleviation consequences caused by excess water (1) (1. At the time of heavy rains Cibinong subdistrict, there are many locations that flood the roads by the water. As it happens precisely at Jalan Pabuaran area Pabuaran there are puddles caused by malfunction of the drainage channel with optimal because the clogged drainage channels and also does not function properly. According Suripin (2) in his book entitled Sustainable Urban Drainage System, drainage has the meaning drain, drain, remove, or diverting water. In general, the drainage water is defined as a series of buildings which serves to reduce or remove the excess water out of an area or land, so the land can function optimally.

Identification of the problem of the small dimension of the channel or not well ordered resulted not able to drain rainwater during heavy rainfall. Overflow of water during the rains, resulting in a puddle on a certain plateau as in Jl.Pabuaran. Terjadinya blockage of drainage channels due to siltation. The purpose of this study is Knowing the value of discharge runoff in drainage channels already ada. Mengevaluasi existing drainage channels to accommodate and drain discharge planning runoff. Mengetahui whether or not a new drainage channel dimensions in Pabuaran road.

(C) The Author(s). 2020 Open Access. This article is distributed under the terms of the Creative Commons Attribution 4.0 International License (http://creativecommons.org/licenses/by/4.0/), which permits unrestricted use, distribution, and reproduction in any medium, provided you give appropriate credit to the original author(s) and the source, provide a link to the Creative Commons license, and indicate if changes were made. 


\section{Volume 04 Number 01 September 2020 METHODOLOGY}

Research The method of this research with case study approach, where the method used is descriptive quantitative and analytical aims to evaluate the condition of a given period as a basis for planning for the future based on the data collected, based on analysis of theoretical and empirical then pulled conclusion of results analysis that has been done.

Hydrological survey conducted to obtain data on hydrology, where the data relate to employment or hydrological analysis is used as a reference in planning capacity of drainage on Jalan Pabuaran Cibinong excl. The hydrological data needed to analyze hydrological, among others:

\section{1 hydrological analysis}

Hydrological data analysis to rainfall, there are several stages to achieve an optimal result. Before the stage of the analysis is done, firstly necessary supporting data that can help the process of analysis.

\section{a. Rainfall intensity}

The intensity of rainfall is the large amount of rain which is expressed in high rainfall or rainfall volume per unit time. The rainfall varies, depending on the duration and frequency of rainfall data processing it happened. For Rainfall intensity Rainfall be used statistical method of observation data duration of rainfall that occurred. If the data for each of rainfall data does not exist, it is necessary to empirically approach the maximum daily rainfall that occur each year.

\section{b. Frequency analysis}

Frequency analysis is a term that refers to the technique of analyzing the probability of occurrence of hydrological variables in the statistical scope (Ponce, 1989). This analysis is needed to determine the return period flood discharge with a specific plan. Of rainfall on average obtained from various stati

ons in the watershed is analyzed statistically to obtain the distribution pattern of rainfall data in accordance with the distribution pattern of rainfall data on average In fact that not all variants of a variable hydrological located or equal to the average value. Variation or dispersion is the high degree of leverage variance around the average value. How to measure the magnitude of dispersion is called dispersion measurement.

\section{c. Analysis of flood discharge}

To calculate how much of the maximum flood discharge that occurred on the road Pabuaran can use some methods, the scientific paper, the author uses two methods to determine the maximum flood discharge, which is a synthetic unit hydrograph Nakayasu and synthetic unit hydrograph ITB.

\subsection{Analysis Hydraulics}

Hydraulics is the study of the properties of liquids and examinations to obtain the formulas and laws liquid in equilibrium (stationary) and in motion. Hydraulics analysis sought to determine the flow capacity of the channel on the current condition of the flood plan from the previous studies and observations obtained. Hydraulics analysis performed on all channels to obtain the desired channel dimensions, namely the water level along the flow channel being reviewed.

\section{a. Existing Drainage Channel Capacity Calculations}

From the analysis of rainfall data processing calculations that have been obtained and are known in the maximum flood discharge Pabuaran way, we then analyzed the volume calculation on drainage channels in accordance with the hydrological data that has been obtained. 
b. New Drainage Channel Capacity Calculations

\section{Volume 04 Number 01 September 2020}

From the analysis of rainfall data processing calculations that have been obtained and are known in the maximum flood discharge Pabuaran way, we then do a comparison analysis of the calculation of the volume of existing drainage channels with the calculation of a new drainage channel in accordance with the hydrological data that has been obtained.

\section{RESULTS AND DISCUSSION}

The calculation below is the discharge of rain water during the 5 years is as follows:

3.1 hydrological analysis

a. Rainfall intensity
$\mathrm{I}=\left(\frac{129.59}{24}\right) \cdot\left(\frac{24}{1}\right)^{\frac{2}{3}}=44.93 \mathrm{~mm} / \mathrm{jam}$

Table 1 Maximum Daily Rainfall Data / year ( $\mathrm{mm} /$ day)

\begin{tabular}{|c|c|c|c|c|c|c|}
\hline & $\mathbf{R 2}$ & R5 & R10 & R25 & R50 & R100 \\
\hline & 112,16 & 129,59 & 140,55 & 153,90 & 163,66 & 173,20 \\
\hline 1 & 38,88 & 44,93 & 48,73 & 53,35 & 56,74 & 60,04 \\
\hline 2 & 24,50 & 28,30 & 30,70 & 33,61 & 35,74 & 37,83 \\
\hline 3 & 18,69 & 21,60 & 23,43 & 25,65 & 27,28 & 28,87 \\
\hline 4 & 15,43 & 17,83 & 19,34 & 21,17 & 22,52 & 23,83 \\
\hline 5 & 13,30 & 15,36 & 16,66 & 18,25 & 19,40 & 20,54 \\
\hline 6 & 11,78 & 13,61 & 14,76 & 16,16 & 17,18 & 18,18 \\
\hline 7 & 10,63 & 12,28 & 13,32 & 14,58 & 15,50 & 16,41 \\
\hline 8 & 9,72 & 11,23 & 12,18 & 13,34 & 14,18 & 15,01 \\
\hline 9 & 8,99 & 10,38 & 11,26 & 12,33 & 13,11 & 13,88 \\
\hline 10 & 8,38 & 9,68 & 10,50 & 11,49 & 12,22 & 12,94 \\
\hline 11 & 7,86 & 9,08 & 9,85 & 10,79 & 11,47 & 12,14 \\
\hline 12 & 7,42 & 8,57 & 9,30 & 10,18 & 10,82 & 11,46 \\
\hline 13 & 7,03 & 8,13 & 8,81 & 9,65 & 10,26 & 10,86 \\
\hline 14 & 6,69 & 7,73 & 8,39 & 9,18 & 9,77 & 10,34 \\
\hline 15 & 6,39 & 7,39 & 8,01 & 8,77 & 9,33 & 9,87 \\
\hline 16 & 6,12 & 7,08 & 7,67 & 8,40 & 8,94 & 9,46 \\
\hline 17 & 5,88 & 6,80 & 7,37 & 8,07 & 8,58 & 9,08 \\
\hline 18 & 5,66 & 6,54 & 7,09 & 7,77 & 8,26 & 8,74 \\
\hline 19 & 5,46 & 6,31 & 6,84 & 7,49 & 7,97 & 8,43 \\
\hline 20 & 5,28 & 6,10 & 6,61 & 7,24 & 7,70 & 8,15 \\
\hline 21 & 5,11 & 5,90 & 6,40 & 7,01 & 7,45 & 7,89 \\
\hline 22 & 4,95 & 5,72 & 6,21 & 6,80 & 7,23 & 7,65 \\
\hline 23 & 4,81 & 5,56 & 6,02 & 6,60 & 7,02 & 7,42 \\
\hline 24 & 4,67 & 5,40 & 5,86 & 6,41 & 6,82 & 7,22 \\
\hline
\end{tabular}

Source: http://dataonline.bmkg.go.id/data_iklim on

b. frequency analysis

Following the calculation of the probability distribution of rainfall with Log Person III. 
Volume 04 Number 01 September 2020

Table 2 Distribution Selection Parameters

\begin{tabular}{|c|c|c|c|}
\hline Distribution Type & Criteria & Score & Information \\
\hline Normal & $\mathrm{Cs}=0$ & 0,8573 & not fulfilled \\
\hline & $\mathrm{Cv}=3$ & 0,1945 & not fulfilled \\
\hline Log Normal & $\begin{array}{c}\mathrm{Cs}=3 \mathrm{C} v+\mathrm{Cv}^{\wedge} 3= \\
0.1412\end{array}$ & 0,3440 & not fulfilled \\
\hline & $\begin{array}{c}\mathrm{Ck}=\mathrm{Cv} 8+6 \mathrm{Cv} 6+ \\
15 \mathrm{Cv} 4+16 \mathrm{Cv}+3\end{array}$ & 4,6195 & not fulfilled \\
\hline Gumbel & $\mathrm{Cs}=1,14$ & 0,8573 & not fulfilled \\
\hline & $\mathrm{Ck}=5,40$ & 5,5010 & not fulfilled \\
\hline Log Pearson Type III & $\mathrm{Cs} \neq 0$ & 0,3440 & fulfilled \\
\hline & Atau selain nilai diatas & 0,0348 & fulfilled \\
\hline
\end{tabular}

Source: Analysis Calculation

c. Analysis of flood discharge

Analysis of Flood Discharge hydrograph Unit Plan with Nakayasu and ITB

Unit hydrograph Nakayasu.

1. Unit hydrograph equation Nakayasu

$$
Q p=\frac{c . A \cdot R o}{3,6\left(0.3 T p+T_{0.3}\right)}
$$

Rainwater flow calculations using formulas Hidrogran Nakayasu unit, the following is a calculation of rain water flow rate for channels $A$, is as follows:

Parameters - parameters required in the calculation as follows:

A channel characteristics include:
Luas Line (A total)
Channel Length $(\mathrm{L})$
$=0.33679 \mathrm{~km}$
$=0.42567 \mathrm{~km} 2$

A channel characteristic coefficient $(\alpha)=2$

Netto rain Unit (Ro) $\quad=1 \mathrm{~mm} /$ hour

Hydrograph parameters include:

$$
\begin{aligned}
\text { Time concentration }(\mathrm{Tg}) & =0,4+0,058 \mathrm{~L} \\
& =0.4+0.058 .0 .42567 \\
& =0425 \text { hours } \\
\text { Rain Length Standard (Tr) } & =0.75 \mathrm{Tg} \\
& =0.75 \times 0.425 \\
& =0319 \text { hours } \\
& =\mathrm{A} \times \mathrm{Tg} \\
& =2 \times 0.425 \\
\text { T } 0.3 & =0849 \text { hours } \\
\text { Concentration time (Tp) } & =\mathrm{Tg}+0.8 \mathrm{Tr} \\
& =0.425+0.8 \times 0.319 \\
& =0680 \text { hour }
\end{aligned}
$$

The peak discharge (Qp)

$Q p=\frac{c . A \cdot R o}{3,6\left(0.3 T p+T_{0.3}\right)}=\frac{1 \cdot 0.33679 .1}{3,6(0.3 \cdot 0 \cdot 680+0.849)}=0.089 \mathrm{~m}^{3} / \mathrm{det}$

Basic flow $(\mathrm{Qb})$

$Q b=0.475 \times A .6444 \times D .9435$

$Q b=0.475 \times 0.336790,6444 \times 0.42567 / 0.336790,9435$

$Q b=0294 \mathrm{~m} 3 / \mathrm{s}$ 
Synthetic unit hydrograph curve equation is:

a. Curved section of the ride for $0 \leq t \leq T p$

$$
Q \alpha=Q p=\left\lceil\frac{t}{T p}\right]^{2.4}
$$

\begin{tabular}{|l|l|}
\hline$t(h)$ & $Q(m 3 / s)$ \\
\hline 0 & 0,000 \\
\hline 0.2 & 0,005 \\
\hline 0.4 & 0,025 \\
\hline .680 & 0.089 \\
\hline
\end{tabular}

b. Curved section down

1. To $Q d>Q p 0.3$ to $T p \leq t \leq T p+T 0,3$

$Q_{p}=Q_{p} \cdot 0.3\left(\frac{\left(t-T_{p}\right)}{T_{0.3}}\right.$

\begin{tabular}{|l|l|}
\hline$t(h)$ & $Q(m 3 / s)$ \\
\hline 1 & 0.056 \\
\hline 1.2 & 0,042 \\
\hline 1.4 & 0,032 \\
\hline 1,529 & 0,027 \\
\hline
\end{tabular}

2. To $0.3 \mathrm{Qp}>\mathrm{Qd}>0.32 \mathrm{Qp}$ to $\mathrm{T} 0,3 \leq \mathrm{t} \leq \mathrm{Tp}+1.5+\mathrm{T} 0,3 \mathrm{~T} 0,3$

$$
Q_{p}=Q_{p} \cdot 0.3\left(\frac{\left(t-T_{p}\right)+\left(0.5-T_{0.3}\right)}{1.5 T_{0.3}}\right.
$$

\begin{tabular}{|l|l|}
\hline$t(h)$ & $Q(\mathrm{~m} 3 / \mathrm{s})$ \\
\hline 2 & .0171 \\
\hline 2.2 & .0141 \\
\hline 2.4 & .0117 \\
\hline 2.6 & .0097 \\
\hline 2.8 & .0080 \\
\hline 2.802946476 & .0080 \\
\hline
\end{tabular}

3. To $0.32 \mathrm{Qp}>\mathrm{Qd}$ for $\mathrm{t} \geq \mathrm{Tp}+1.5+\mathrm{T} 0,3 \mathrm{~T} 0,3$

$Q_{p}=Q_{p} .0 .3\left(\frac{\left(t-T_{p}\right)+\left(0.5-T_{0.3}\right)}{2 T_{0.3}}\right.$ 


\section{Volume 04 Number 01 September 2020}

\begin{tabular}{|l|l|}
\hline $\mathrm{t}(\mathrm{h})$ & $\mathrm{Q}(\mathrm{m} 3 / \mathrm{s})$ \\
\hline 3 & 0.006952 \\
\hline 4 & 0.003422 \\
\hline 5 & 0.001685 \\
\hline 6 & 0.000829 \\
\hline 7 & 0.000408 \\
\hline 8 & 0.000201 \\
\hline 9 & 0.000099 \\
\hline 10 & 0.000049 \\
\hline 11 & 0.000024 \\
\hline 12 & 0.000012 \\
\hline 13 & 0.000006 \\
\hline 14 & 0.000003 \\
\hline 15 & 0.000001 \\
\hline 16 & 0.000001 \\
\hline 17 & 0.000000 \\
\hline 18 & 0.000000 \\
\hline 19 & 0.000000 \\
\hline 20 & 0.000000 \\
\hline 21 & 0.000000 \\
\hline 22 & 0.000000 \\
\hline 23 & 0.000000 \\
\hline 24 & 0.000000 \\
\hline
\end{tabular}

Debit recapitulation Nakayasu flood hydrograph Plan:

Table 3 Recapitulation Debit flood hydrograph Plan Nakayasu

\begin{tabular}{|l|l|l|l|l|l|l|l|}
\hline \multirow{2}{*}{$\mathrm{t}(\mathrm{h})$} & $\mathrm{Q}(\mathrm{m} 3 /$ & \multicolumn{6}{|c|}{ Debit magnitude of each period $(\mathrm{m} 3 / \mathrm{s})$} \\
\cline { 3 - 8 } & $\mathrm{s})$ & $\mathrm{R} 2$ & $\mathrm{R} 5$ & $\mathrm{R} 10$ & $\mathrm{R} 25$ & $\mathrm{R} 50$ & $\mathrm{R} 100$ \\
\hline 0 & 0,000 & .294 & .294 & .294 & .294 & .294 & .294 \\
\hline 0.2 & 0,005 & 0.529 & .566 & .589 & 0.617 & .637 & 0.657 \\
\hline 0.4 & 0,025 & 1,596 & 1,799 & 1,926 & 2,081 & 2,194 & 2,305 \\
\hline .680 & 0.089 & 5.087 & 5.832 & 6,301 & 6.871 & 7.288 & 7.696 \\
\hline 1 & 0.056 & 4.516 & 5.173 & 5.585 & 6.088 & 6.455 & 6.814 \\
\hline 1.2 & 0,042 & 4,158 & 4.759 & 5,136 & 5.596 & 5.932 & 6.261 \\
\hline 1.4 & 0,032 & 3.746 & 4.283 & 4,620 & 5.031 & 5,331 & 5.625 \\
\hline 1,529 & 0,027 & 3.374 & 3,852 & 4.153 & 4.519 & 4.787 & 5,050 \\
\hline 2 & 0,017 & 2.433 & 2,765 & 2,974 & 3,229 & 3.415 & 3,597 \\
\hline 2.2 & 0,014 & 1.953 & 2.211 & 2,373 & 2,570 & 2.714 & 2,856 \\
\hline 2.4 & 0,012 & 1,604 & 1,807 & 1.935 & 2,091 & 2.205 & 2.316 \\
\hline 2.6 & 0,010 & 1,343 & 1.506 & 1.609 & 1,733 & 1,825 & 1,914 \\
\hline 2.8 & 0,008 & 1,132 & 1,262 & 1,344 & 1,444 & 1,517 & 1,588 \\
\hline 2,803 & 0,008 & 1.055 & 1.174 & 1,248 & 1.339 & 1.405 & 1,470 \\
\hline 3 & 0.007 & 0.958 & 1,062 & 1.127 & 1.206 & 1,264 & 1,320 \\
\hline 4 & 0,003 & 0.744 & .814 & 0.858 & 0.912 & 0.951 & 0,990 \\
\hline 5 & 0,002 & 0.592 & .639 & 0.668 & 0,703 & 0,729 & 0.755 \\
\hline 6 & 0,001 & .487 & 0,517 & .536 & 0,559 & 0.576 & 0.593 \\
\hline 7 & 0,000 & 0,407 & 0,425 & 0.436 & .450 & .460 & 0.469 \\
\hline
\end{tabular}




\begin{tabular}{|l|l|l|l|l|l|l|l|}
\hline \multicolumn{7}{|c|}{ Volume 04 Number 01 September 2020 } \\
\hline \multirow{2}{*}{$\mathrm{t}(\mathrm{h})$} & $\mathrm{Q}(\mathrm{m} 3 /$ & \multicolumn{7}{c|}{ Debit magnitude of each period (m3 / s) } \\
\cline { 3 - 8 } & $\mathrm{s})$ & $\mathrm{R} 2$ & $\mathrm{R} 5$ & $\mathrm{R} 10$ & $\mathrm{R} 25$ & $\mathrm{R} 50$ & $\mathrm{R} 100$ \\
\hline 8 & 0,000 & .350 & 0.358 & 0.364 & .371 & 0,375 & .380 \\
\hline 9 & 0,000 & 0.321 & .326 & 0.328 & 0.332 & 0.334 & .336 \\
\hline 10 & 0,000 & 0.307 & .309 & 0,311 & 0,312 & 0.314 & 0.315 \\
\hline 11 & 0,000 & 0.301 & 0,302 & 0,302 & 0,303 & 0.304 & 0.304 \\
\hline 12 & 0,000 & 0.297 & .298 & .298 & .298 & 0,299 & 0,299 \\
\hline 13 & 0,000 & 0.295 & 0,296 & 0,296 & 0,296 & 0,296 & 0,296 \\
\hline 14 & 0,000 & 0.295 & 0.295 & 0.295 & 0.295 & 0.295 & 0.295 \\
\hline 15 & 0,000 & .294 & .294 & .294 & .294 & .294 & .294 \\
\hline 16 & 0,000 & .294 & .294 & .294 & .294 & .294 & .294 \\
\hline 17 & 0,000 & .294 & .294 & .294 & .294 & .294 & .294 \\
\hline 18 & 0,000 & .294 & .294 & .294 & .294 & .294 & .294 \\
\hline 19 & 0,000 & .294 & .294 & .294 & .294 & .294 & .294 \\
\hline 20 & 0,000 & .294 & .294 & .294 & .294 & .294 & .294 \\
\hline 21 & 0,000 & .294 & .294 & .294 & .294 & .294 & .294 \\
\hline 22 & 0,000 & .294 & .294 & .294 & .294 & .294 & .294 \\
\hline 23 & 0,000 & .294 & .294 & .294 & .294 & .294 & .294 \\
\hline 24 & 0,000 & .294 & .294 & .294 & .294 & .294 & .294 \\
\hline
\end{tabular}

\section{DEBIT BANJIR HIDROGRAF SATUAN SINTETIK METODE NAKAYASU}

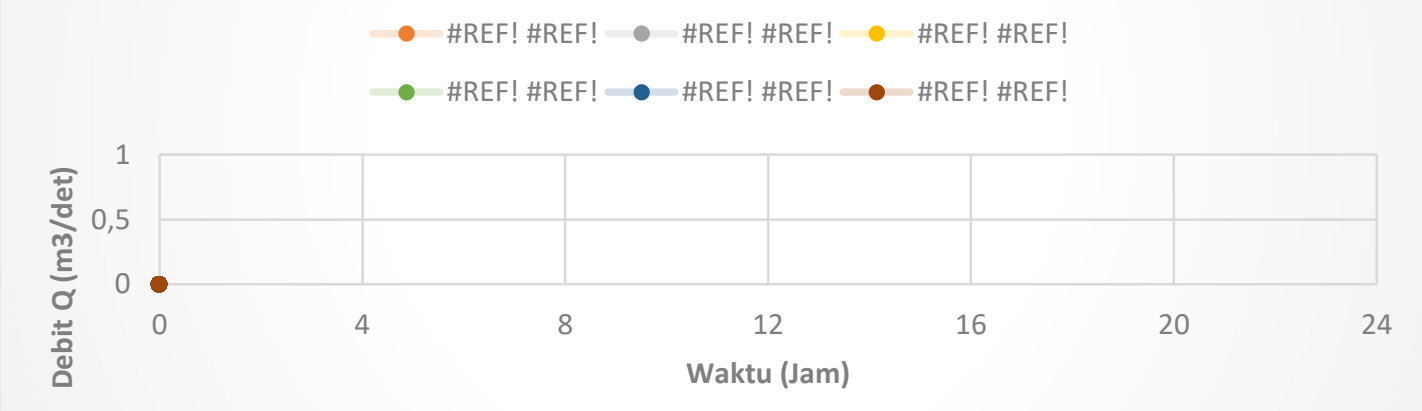

Figure 1 Debit flood hydrograph Unit Synthetic Methods Nakayasu Source: Analysis Calculation

2. Hydrograph equation ITB unit

1. Synthetic Unit Hydrograph equation ITB $Q_{p}=\frac{R}{3.6 T_{p}} \frac{A_{D A S}}{A_{H S S}}$

2. Parameters - parameters required in the calculation as follows:

Watershed characteristics include:

$\begin{array}{ll}\text { Size Channel } 7 \text { (A total) } & =0.33679 \mathrm{~km} \\ \text { Channel Length }(\mathrm{L}) & =0.42567 \mathrm{~km}\end{array}$




\section{Volume 04 Number 01 September 2020}

High Effective Precipitation $(\mathrm{R})=1 \mathrm{~mm} / \mathrm{hr}$

Duration of rainfall unit $(\mathrm{Tr}) \quad=1$ hour

The coefficient of time $(\mathrm{Ct}) \quad=1$

Run Off coefficient $(\mathrm{Cp}) \quad=1$

Alpha $\quad=1$

Size AhSS $\quad=1,121$

Hydrograph parameters include:

TL

$=\mathrm{Ct} \times 0.18225 \times \mathrm{L} 0.6$

$=1 \times 0.18225 \times 0.425670,6$

$=0.109172$ hours

Concentration time $(\mathrm{Tp}) \quad=\mathrm{TL}+0.5 \mathrm{Tr}$

$$
\begin{aligned}
& =0.109172+0.5 \times 1 \\
& =0.609172 \text { hours }
\end{aligned}
$$

The peak discharge (Qp)

$Q_{p}=\frac{R}{3.6 T_{p}} \frac{A_{D A S}}{A_{H S S}}=\frac{1}{3.6 .0 .609172} \frac{0.33679}{1.121}=0.137$

Basic flow $(\mathrm{Qb})$

$Q b=0.475 \times A^{.6444} x D^{.9435}$

$Q b=0.475 \times 0.33679 \cdot 6444 x 0.42567 / 0.33679 \cdot 9435$

$Q b=0294^{\mathrm{m} 3} / \mathrm{sec}$

3. Synthetic Unit hydrograph flood discharge ITB:

Table 4 Debit flood hydrograph Synthetic ITB

\begin{tabular}{|c|c|c|c|c|}
\hline \multirow{2}{*}{$\mathbf{T}(\mathbf{h})$} & $\mathbf{t}$ & $\mathbf{Q}$ & \multirow{2}{*}{$\mathbf{A}$} & \multirow{2}{*}{$\mathbf{Q}$} \\
\cline { 2 - 3 } & $\mathbf{T} / \mathbf{T p}$ & $\mathbf{Q} / \mathbf{Q p}$ & & \\
\hline 0 & 0,000 & 0.0000 & 0,000 & 0,000 \\
\hline 1 & 1,642 & .7782 & .6388 & .1066 \\
\hline 2 & 3.283 & .2044 & .3355 & .0280 \\
\hline 3 & 4.925 & .0438 & .1079 & 0.0060 \\
\hline 4 & 6.566 & .0089 & .0293 & 0.0012 \\
\hline 5 & 8.208 & 0.0018 & .0073 & 0.0002 \\
\hline 6 & 9.849 & 0.0004 & 0.0017 & 0.0000 \\
\hline 7 & 11.491 & 0.0001 & 0.0004 & 0.0000 \\
\hline 8 & 13.133 & 0.0000 & 0.0001 & 0.0000 \\
\hline 9 & 14.774 & 0.0000 & 0.0000 & 0.0000 \\
\hline 10 & 16.416 & 0.0000 & 0.0000 & 0.0000 \\
\hline 11 & 18.057 & 0.0000 & 0.0000 & 0.0000 \\
\hline 12 & 19.699 & 0.0000 & 0.0000 & 0.0000 \\
\hline 13 & 21.340 & 0.0000 & 0.0000 & 0.0000 \\
\hline
\end{tabular}




\section{Volume 04 Number 01 September 2020}

\begin{tabular}{|c|c|c|c|c|}
\hline 14 & 22,982 & 0.0000 & 0.0000 & 0.0000 \\
\hline 15 & 24.624 & 0.0000 & 0.0000 & 0.0000 \\
\hline 16 & 26.265 & 0.0000 & 0.0000 & 0.0000 \\
\hline 17 & 27.907 & 0.0000 & 0.0000 & 0.0000 \\
\hline 18 & 29.548 & 0.0000 & 0.0000 & 0.0000 \\
\hline 19 & 31.190 & $2,0 \mathrm{E}-13$ & 0.0000 & 0.0000 \\
\hline 20 & 32.831 & $4,0 \mathrm{E}-14$ & 0.0000 & $5,4 \mathrm{E}-15$ \\
\hline 21 & 34.473 & $7,7 \mathrm{E}-15$ & 0.0000 & $1,1 \mathrm{E}-15$ \\
\hline 22 & 36.115 & $1,5 \mathrm{E}-15$ & $2,6844 \mathrm{E}-14$ & $2,0 \mathrm{E}-16$ \\
\hline 23 & 37.756 & $2,9 \mathrm{E}-16$ & $5,44189 \mathrm{E}-15$ & $3,9 \mathrm{E}-17$ \\
\hline 24 & 39.398 & $5,6 \mathrm{E}-17$ & $1,101 \mathrm{E}-15$ & $7,7 \mathrm{E}-18$ \\
\hline
\end{tabular}

Source: Analysis Calculation

Recapitulation of the flood discharge plan Synthetic Unit Hydrograph ITB:

Table 5 Recapitulation Debit flood hydrograph Plan Synthetic ITB

\begin{tabular}{|c|c|c|c|c|c|c|c|}
\hline \multirow{2}{*}{$\mathrm{t}(\mathrm{h})$} & \multirow{2}{*}{$\mathrm{Q}(\mathrm{m} 3 / \mathrm{s})$} & \multicolumn{6}{|c|}{ Debit magnitude of each period (m3/s) } \\
\cline { 3 - 7 } & & $\mathrm{R} 2$ & $\mathrm{R} 5$ & $\mathrm{R} 10$ & $\mathrm{R} 25$ & $\mathrm{R} 50$ & $\mathrm{R} 100$ \\
\hline 0 & 0,000 & 0,000 & 0,000 & 0,000 & 0,000 & 0,000 & 0,000 \\
\hline 1 & 0.107 & 5.608 & 6.434 & 6.954 & 7.586 & 8.048 & 8.501 \\
\hline 2 & 0,028 & 3,071 & 3.503 & 3,774 & 4,104 & 4.346 & 4.582 \\
\hline 3 & 0,006 & 1,925 & 2,178 & 2,338 & 2,532 & 2,674 & 2.812 \\
\hline 4 & 0,001 & 1,458 & 1,639 & 1,753 & 1.892 & 1,993 & 2,092 \\
\hline 5 & 0,000 & 1,230 & 1,376 & 1,468 & 1,579 & 1,660 & 1,740 \\
\hline 6 & 0,000 & 0.525 & .561 & .584 & 0.611 & .631 & 0.651 \\
\hline 7 & 0,000 & 0.343 & .350 & 0.355 & 0.361 & 0,365 & .369 \\
\hline 8 & 0,000 & 0.304 & 0,305 & 0.306 & 0.307 & 0,308 & .309 \\
\hline 9 & 0,000 & 0,296 & 0,296 & 0,296 & 0.297 & 0.297 & 0.297 \\
\hline 10 & 0,000 & .294 & .294 & .294 & .294 & .294 & .294 \\
\hline 11 & 0,000 & .294 & .294 & .294 & .294 & .294 & .294 \\
\hline 12 & 0,000 & .294 & .294 & .294 & .294 & .294 & .294 \\
\hline 13 & 0,000 & .294 & .294 & .294 & .294 & .294 & .294 \\
\hline 14 & 0,000 & .294 & .294 & .294 & .294 & .294 & .294 \\
\hline 15 & 0,000 & .294 & .294 & .294 & .294 & .294 & .294 \\
\hline 16 & 0,000 & .294 & .294 & .294 & .294 & .294 & .294 \\
\hline 17 & 0,000 & .294 & .294 & .294 & .294 & .294 & .294 \\
\hline 18 & 0,000 & .294 & .294 & .294 & .294 & .294 & .294 \\
\hline 19 & 0,000 & .294 & .294 & .294 & .294 & .294 & .294 \\
\hline 20 & 0.0000 & .294 & .294 & .294 & .294 & .294 & .294 \\
\hline 21 & $1,05 \mathrm{E}-15$ & .294 & .294 & .294 & .294 & .294 & .294 \\
\hline 22 & $2,04 \mathrm{E}-16$ & .294 & .294 & .294 & .294 & .294 & .294 \\
\hline 23 & $3,95 \mathrm{E}-17$ & .294 & .294 & .294 & .294 & .294 & .294 \\
\hline 24 & $7,66 \mathrm{E}-18$ & .294 & .294 & .294 & .294 & .294 & .294 \\
\hline
\end{tabular}


Volume 04 Number 01 September 2020

\section{DEBIT BANJIR HIDROGRAF SATUAN SINTETIK METODE ITB}

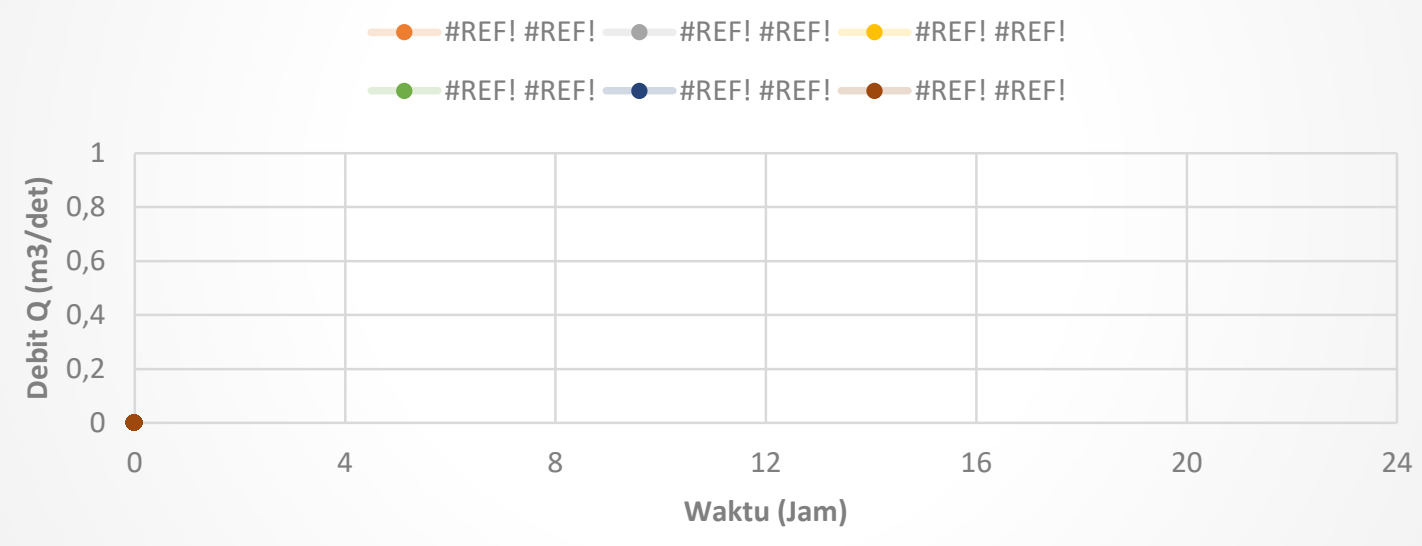

Figure 2 Chart Debit flood hydrograph Unit Synthetic ITB

Source: Analysis Calculation

Recapitulation Debit flood hydrograph Plan Synthetic ITB

\begin{tabular}{|c|c|c|}
\hline \multirow{2}{*}{$\begin{array}{l}\text { Periode } \\
\text { T tahun }\end{array}$} & \multicolumn{2}{|c|}{ Debit Maksimum (m3/det) } \\
\hline & Nakayasu & ITB \\
\hline 2 & 5.087 & 5,608 \\
\hline 5 & 5,832 & 6,434 \\
\hline 10 & 6,301 & 6,954 \\
\hline 25 & 6,871 & 7,586 \\
\hline 50 & 7,288 & 8,048 \\
\hline 100 & 7,696 & 8,501 \\
\hline
\end{tabular}

Source: Analysis Calculation

3.2 analysis Hydraulics

a. Dimension calculation Existing Channels

Here is the calculation for the channel A.

$\mathrm{b}=0,92 \mathrm{~m}$

$\mathrm{h}=0,65 \mathrm{~m}$

$\mathrm{S}=0.014$

Cross-sectional area $(A)$ :

$\mathrm{A}=\mathrm{b} \times \mathrm{h}$

$A=0,92 \times 0,65=0,598 \mathrm{~m}^{2}$

Wet circumference $(P)$

$\mathrm{P}=\mathrm{b}+2 \mathrm{~h}$

$\mathrm{P}=0,92+(2 \times 0,65)=2.22 \mathrm{~m}$

Finger - the finger hydraulic $(R)$ :

$\mathrm{R}=\frac{\mathrm{A}}{\mathrm{P}}$

$\mathrm{R}=\frac{0,598}{2.22}=0,269 \mathrm{~m}$

The flow velocity $(\mathrm{V})$; 


$$
\begin{aligned}
& \mathrm{n}=0,014 \\
& \mathrm{~V}=\frac{1}{\mathrm{n}} \mathrm{R}^{\frac{2}{3}} \mathrm{~S}^{\frac{1}{2}} \\
& \mathrm{~V}=\frac{1}{0,014} 0,269^{\frac{2}{3}} \cdot 0,014^{\frac{1}{2}}=3.51 \mathrm{~m} / \mathrm{dt} \\
& \mathrm{Q}_{\text {sal }}=\mathrm{A} \times \mathrm{V}=0,598 \times 3.51=2.1 \mathrm{~m}^{3} / \mathrm{dt}
\end{aligned}
$$

Volume 04 Number 01 September 2020

\begin{tabular}{|c|c|c|c|c|c|c|c|c|c|c|c|}
\hline No. & b & $\mathrm{h}$ & \multirow{2}{*}{ S } & A & $P$ & $\mathrm{R}$ & \multirow{2}{*}{$\mathrm{n}$} & V & Qsal & Qaliran & \multirow{2}{*}{ keterangar } \\
\hline Saluran & (m) & (m) & & (m2) & (m) & (m) & & $(\mathrm{m} / \mathrm{dt})$ & (m3/det) & (m3/det) & \\
\hline A & 0,92 & 0,65 & 0,014 & 0,60 & 2,22 & 0,27 & 0.014 & 3,51 & 2,10 & 6,434 & Limpas \\
\hline$B$ & 0,43 & 0,25 & 0,014 & 0,11 & 0,93 & 0,12 & 0.014 & 1,99 & 0,21 & 6.434 & Limpas \\
\hline
\end{tabular}

If $Q$ plan $<Q$ channel, So the drainage channel able to accommodate the flow of flood discharge, and declared safe. For channel B measures the same calculation as channel A.

Table 6 Calculation Existing Channel Dimensions

Source: Analysis Calculation
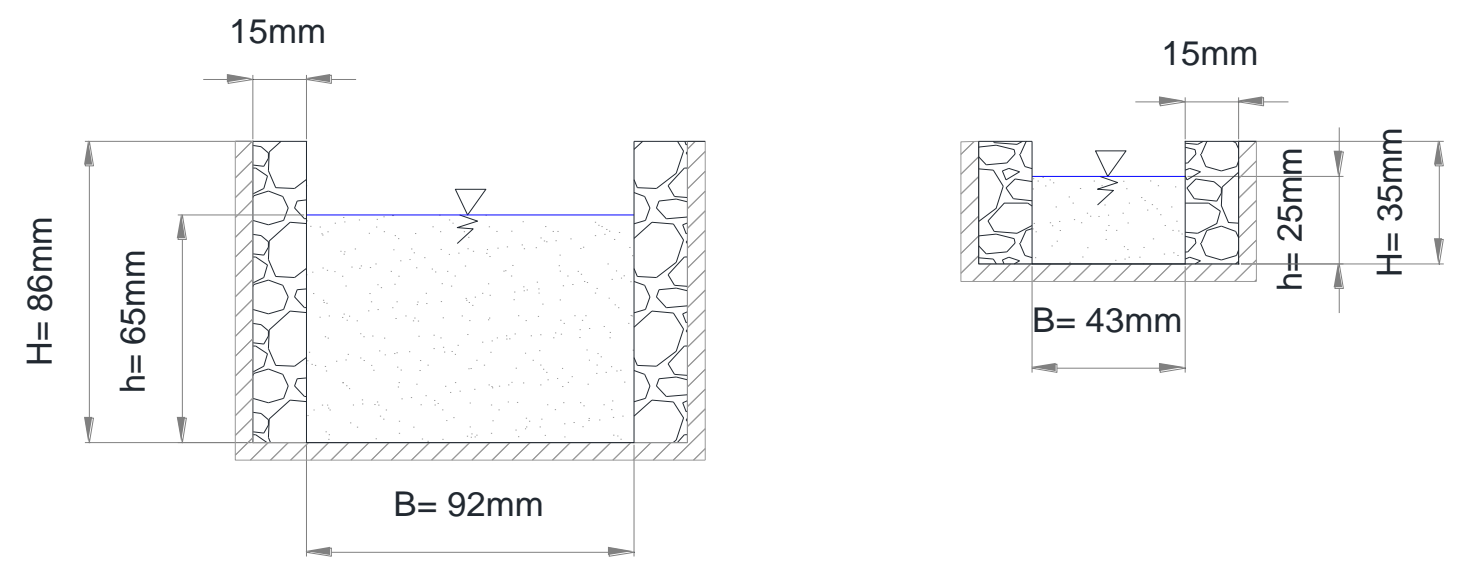

\section{Existing Channels Figure 3 Dimensions Source: Analysis Calculation}

b. Calculations Dimension New Channel

Calculations are based on the discharge channel dimensions that must be accommodated by the channel (Qs in $\mathrm{m}^{3} / \mathrm{s}$ ) greater than or equal to the discharge plan caused by rain plan (QT in $\mathrm{m}^{3} / \mathrm{s}$ ). Such conditions can be formulated by the following equation: Qs $\geq$ QT. Next will be the calculation of a new dimension to insecure channels, to determine the dimensions of the safe. Determination of the drainage channel dimensions done by trial and error or trial and error.

Information :

$A=$ wet cross-sectional area $(\mathrm{m} 2)$

$P=$ circumference of wet flow $(m)$

$\mathrm{R}=$ hydraulic radius $(\mathrm{m})$

$\mathrm{V}=$ flow velocity $(\mathrm{m} / \mathrm{sec})$

$\mathrm{W}=$ high surveillance $(\mathrm{m})$

$\mathrm{b}=$ width of the base line $(\mathrm{m})$

$\mathrm{y}=$ high water level $(\mathrm{m})$

$\mathrm{H}=$ height of the channel $(\mathrm{m})$ 
$\mathrm{n}=$ roughness manning

$S=$ slope of the channel base

calculation:

Cross-sectional area $(A)$ :

$\mathrm{A}=\mathrm{b} \times \mathrm{h}$

$\mathrm{A}=1.3 \times 1.00=1.30 \mathrm{~m}^{2}$

Wet circumference $(P)$

$\mathrm{P}=\mathrm{b}+2 \mathrm{~h}$

$\mathrm{P}=1.3+(2 \times 1)=2.8 \mathrm{~m}$

Finger - the finger hydraulic $(R)$ :

$\mathrm{R}=\frac{\mathrm{A}}{\mathrm{P}}$

$\mathrm{R}=\frac{1.3}{2.8}=0,46 \mathrm{~m}$

The flow velocity $(\mathrm{V})$;

$\mathrm{n}=0,014$

$\mathrm{V}=\frac{1}{\mathrm{n}} \mathrm{R}^{\frac{2}{3}} \mathrm{~S}^{\frac{1}{2}}$

$\mathrm{V}=\frac{1}{0,014} 0,46^{\frac{2}{3}} \cdot 0,014^{\frac{1}{2}}=5.05 \mathrm{~m} / \mathrm{dt}$

Calculation of discharge channel plans (Q) located in the area around JI Pabuaran Cibinong District of Bogor Regency can be calculated using the following formula:

controls:

$\mathrm{Q}_{\text {sal }}=\mathrm{AxV}=1.305 .05=6.57 \mathrm{~m}^{3} / \mathrm{dt}$

Table 7 Debit Relationship with surveillance channel High Wasters

\begin{tabular}{|c|c|c|}
\hline debit Flood & $\begin{array}{c}\text { High dike } \\
\text { surveillance }\end{array}$ & $\begin{array}{c}\text { High } \\
\text { surveillance } \\
\text { Couples }\end{array}$ \\
\hline $\mathbf{( M}^{\mathbf{3}}$ / sec) & (M) & (M) \\
\hline$<0,50$ & 0.4 & 0.2 \\
\hline 0.50 to 1.50 & 0.5 & 0.20 \\
\hline 1.50 to 5.00 & 0.6 & 0.25 \\
\hline 5.00 to 10.00 & 0.75 & 0.30 \\
\hline 10.00 to 15.00 & 0.85 & 0.40 \\
\hline$>15.00$ & 1.00 & $\mathbf{0 . 5 0}$ \\
\hline
\end{tabular}

source: Planning Criteria Section Channel KP-03, Standard

Based on the results of calculating the dimensions of the drainage channel with a channel cross-sectional area $1: 30 \mathrm{~m}^{2}$ wet so that the maximum discharge usually accommodated by drainage channels designed is $6.57 \mathrm{~m}^{3} / \mathrm{s}$. Based on the above calculation, then used a method ITB in 5-year return period was $6,434 \mathrm{~m}^{3} / \mathrm{s}$. Thus these dimensions can be used for drainage channels. (Sustainable Urban Drainage Systems Dr. Ir. Suripin, M.Eng) 
Volume 04 Number 01 September 2020

Dimension Calculation Table 8 New Channel

\begin{tabular}{|c|c|c|c|c|c|c|c|c|c|c|c|}
\hline No. & $\mathrm{b}$ & $\mathrm{h}$ & \multirow{2}{*}{ S } & A & $\mathrm{P}$ & $\mathrm{R}$ & \multirow{2}{*}{$\mathrm{n}$} & V & Qsal & Qaliran & \multirow{2}{*}{ information } \\
\hline Channel & (M) & (M) & & (M2) & (M) & (M) & & $(\mathrm{M} / \mathrm{sec})$ & $(\mathrm{M} 3 / \mathrm{s})$ & $(\mathrm{M} 3 / \mathrm{s})$ & \\
\hline$A$ & 1.3 & 1 & 0,014 & 1.30 & 2.80 & 0.46 & 0014 & 5,05 & 6.57 & 6.434 & secure \\
\hline$B$ & 1.3 & 1 & 0,014 & 1.30 & 2.80 & 0.46 & 0014 & 5,05 & 6.57 & 6.434 & secure \\
\hline
\end{tabular}

Source: Analysis Calculation

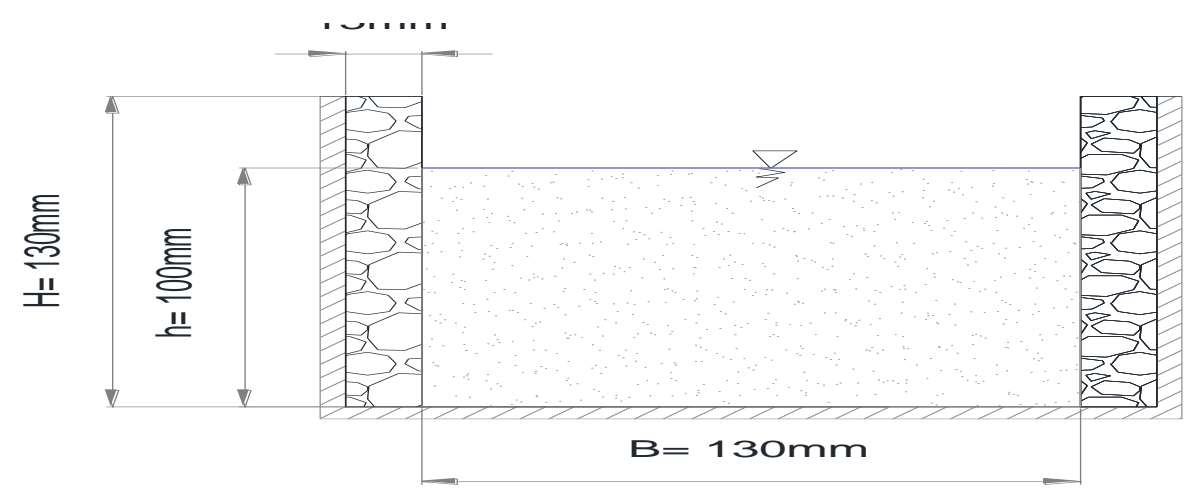

Figure 4 Dimensions New Channel

Source: Analysis Calculation

\section{CONCLUSION}

From the results of research and testing results are as follows:

1. The amount of rainfall in the study site plan for the 5 year return period based on the analysis of Gumbel method amounted to $135.16 \mathrm{~mm} /$ hour.

2. A channel capacity of the existing capacity for discharge by $2: 10 \mathrm{~m}^{3} / \mathrm{sec}$. The capacity of the existing and $B$ channels can accommodate the discharge of $0: 21 \mathrm{~m}^{3} /$ sec

3. 5 year plan flood discharge in Channel A Jalan Pabuaran is $6,434 \mathrm{~m}^{3} / \mathrm{sec}$, while the 5 year plan of flood discharge in Channel B Street Pabuaran is $1,651 \mathrm{~m}^{3} / \mathrm{sec}$

4. The capacity of the existing channels can not accommodate the flood discharge plan. Then it will be normalized, or planning a new channel dimensions.

5. Dimensional channel $A$ beginning sized Width $B=0.92 \mathrm{~m}$ height $\mathrm{H}=0.86 \mathrm{~m}$ and height of water level $h=0.65$ after checking the capacity to accommodate discharge plan then channel $A$ must do change the dimension to width $B=1.3 \mathrm{~m}$ height $\mathrm{H}=1.3$ $\mathrm{m}$ and water level $\mathrm{h}=1 \mathrm{~m}$.

\section{REFERENCE}

[1] suhardjono. drainase. Fakultas Tekhnik UNiversitas Brawijaya ,Malang : s.n., 1984.

[2] suripin. Sistem Drainase Perkotaan yang Berkelanjutan. Yogyakarta : Andi , 2004.

[3] Jurnal Sipil Statik. Pioh, A, H Sumarauw J, S and Mananoma, T. 2019, Tinjauan Sistem Drainase Di Jalan Pelleng,Kleak Kecamatan Malalayang, Kota Manado, pp. 7-9. 
Volume 04 Number 01 September 2020

[4] Jurnal Teknik Sipil. Sulistiono, B and Ardiyanto, A, F. 2017, Evaluasi Kapasitas Saluran Drainase Desa Sariharjo Ngaglik Sleman Yogyakarta, pp. 47-52.

[5] Triatmodjo, Bambang. Hidrologi Terapan. Yogyakarta : Beta Offset, 2008.

[6] Seminar Teknik Sumber Daya Air. Natakusumah, D, K, Hatmoko, W and Harlan, D. Bandung: s.n., 2010, Prosedur Umum Perhitungan HIdrograf Satuan Sintetis (HSS) Untuk Perhitungan Hidrograf Banjir Rencana Studi kasus Pengembangan HSS ITb-1 \& HSS ITB-2.

[7] Wesli. Drainase Perkotaan, Tabel Koefisien Kekasaran Manning. 2008.

[8] S1 Thesis. Topani, Anton. Universitas Mercu Buana, Jakarta: s.n., 2018, Evaluasi Sistem Perencanaan Drainase Pada Jalan Otto Iskandardinata .

[9] soewarno. Hidrologi Aplikasi Metode Statistik Untuk Analisa Data Jilid 1 \& Jilid 2 . Bandung : s.n., 1995.

[10] Kodoatjie, J, R and Sugiyanto. Banjir. 2001 